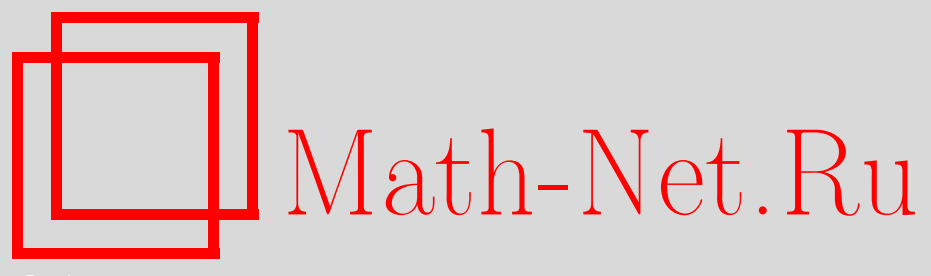

M. Палезе, Е. Уинтеррот, О соотношении между морфизмом Якоби и гессианом в калибровочноестественных теориях поля, ТМФ, 2007, том 152, номер 2, 377-389

DOI: https://doi.org/10.4213/tmf6094

Использование Общероссийского математического портала Math-Net.Ru подразумевает, что вы прочитали и согласны с пользовательским соглашением http://www . mathnet.ru/rus/agreement

Параметры загрузки:

IP: 54.205 .225 .156

26 апреля 2023 г., 11:44:10 


\section{О СООТНОШЕНИИ МЕЖДУ МОРФИЗМОМ ЯКОБИ И ГЕССИАНОМ В КАЛИБРОВОЧНО-ЕСТЕСТВЕННЫХ ТЕОРИЯХ ПОЛЯ}

Полученный Голдшмидтом и Стернбергом классический результат, связывающий морфизм Якоби с гессианом для теорий поля первого порядка, обобщается на калибровочно-естественные теории поля высшего порядка. В частности, определен обобщенный калибровочно-естественный морфизм Якоби, где вариационные векторные поля являются производными Ли сечений калибровочноестественного расслоения по отношению к калибровочно-естественным подъемам инфинитезимальных главных автоморфизмов, и установлена связь с гессианом. Гессиан также очень просто связан с обобщенным морфизмом Бергманна-Бианки, ядро которого дает необходимое и достаточное условие существования глобальных канонических суперпотенциалов. Получено, что гамильтоновы уравнения для гамильтоновой связности, ассоциированной с соответствующим образом определенным ковариантным сильно сохраняющимся током, тождественно удовлетворяются; их можно интерпретировать как обобщенные тождества Бергманна-Бианки и, таким образом, охарактеризовать в терминах обращения гессиана в нуль.

Ключевые слова: струи, калибровочно-естественное расслоение, вторая вариационная производная, обобщенный морфизм Якоби.

\section{1. ВВЕДЕНИЕ}

Несколько формулировок исчисления вариаций на пространстве струй (см., например, работы [1]-[9]) были предложены с целью перевести этот важный аспект математики в область дифференциальной геометрии. Пространства струй в действительности задают естественный контекст для дифференциальных уравнений в дифференциальной геометрии. При такой формулировке оказывается, что дифференциальный оператор, преобразующий лагранжианы в выражения Эйлера-Лагранжа, является не чем иным, как пучковым морфизмом некоторой последовательности пучков [9]-[13], чем определяются два связанных контекста [14]: вариационные бикомплексы бесконечного порядка и вариационные последовательности Крупки конечного порядка. Подход, основанный на струях бесконечного порядка, является во

${ }^{*}$ Department of Mathematics, University of Torino, via C. Alberto 10, 10123 Torino, Italy. E-mail: marcella.palese@unito.it, ekkehart.winterroth@unito.it 
многих отношениях более простым и элегантным, поскольку сразу приводит к результатам, справедливым для любого порядка; рассматривающий конечные порядки подход применяется, когда важным может оказаться сохранение фиксированного порядка, и при этом существенна получающаяся полиномиальная структура вариационных объектов.

В рамках вариационных последовательностей конечного порядка мы характеризуем вторую вариацию калибровочно-естественного инвариантного лагранжиана произвольного порядка в общем случае $n$ независимых переменных и $m$ неизвестных функций и применяем полученный результат для специализации теорем Нётер и соответствующих сохраняющихся токов и суперпотенциалов. В работе [15] Бергманн ввел так называемые обобщенные тождества Бианки для геометрических теорий поля, что позволило получить (после интегрирования по частям) самосогласованное уравнение, включающее локальные дивергенции в первой вариационной формуле.

Хорошо известно, что согласно теории Нётер [16] в классической лагранжевой формулировке теорий поля описание симметрий и сохраняющихся величин состоит в выводе существования подходящих сохраняющихся токов из инвариантности лагранжиана; оказывается, что в наиболее актуальных физических теориях эти токи являются дивергенцией кососимметричных (тензорных) плотностей, которые называются суперпотенииалами для самих сохраняющихся токов. Также хорошо известно, что важность суперпотенциалов определяется возможностью их интегрирования с целью получения сохраняющихся величин, связанных с сохраняющимися токами через теорему Стокса (см., например, работы [17], [18] и ссылки в них). В рамках такой картины обобщенные тождества Бергманна-Бианки являются необходимым и локально достаточным условием для того, чтобы сохраняющиеся нётеровские токи не только были замкнутыми, но и являлись дивергенциями суперпотенциала вдоль решений уравнений Эйлера-Лагранжа. Однако существует проблема общей ковариантности таких тождеств; она уже была поставлена и частично исследована в работе [19], где изучалась инвариантность по отношению к преобразованиям временно́й координаты. Эта проблема, очевидно, отражается на ковариантности сохраняющихся величин.

В ряде работ [20]-[24] мы предложили подход к изучению задачи ковариантности сохраняющихся величин с использованием вариационных производных, взятых по отношению к обобщенным векторным полям, выбранным таким образом, что они представляют собой производные Ли сечений калибровочно-естественных расслоений, перенесенных путем калибровочно-естественных подъемов инфинитезимальных главных автоморфизмов. Такие производные можно подходящим образом интерпретировать как вертикальные дифференциалы [2], [25]. Хорошо известно, что вторая вариация лагранжиана определяется с точностью до полных дивергенций. Мы воспользуемся предыдущими результатами, касающимися второй вариационной производной и морфизма Якоби, полученными в работах [20]-[23], [25], и обобщим классический результат Голдшмидта и Стернберга, связывающий гессиан с морфизмом Якоби для теорий поля первого порядка, на калибровочно-естественные теории поля высшего порядка. Теорема 2 связывает морфизм Якоби с гессианом, 
а теорема 3 определяет их связь с морфизмом Бергманна-Бианки. Мы воспроизводим и уточняем результаты, уже полученные для поля теорий высшего порядка в рамках “факторизованной” вариационной последовательности, где дивергенции были факторизованы априори [25].

В разделе 2 дается конструктивное определение калибровочно-естественных расслоений и указывается на некоторые важные свойства производной Ли сечений таких расслоений (предполагается, что они представляют физические поля). В разделе 3 мы напоминаем подход, основанный на вариационных последовательностях, представляем теоремы Нётер, вводим обобщенный калибровочно-естественный морфизм Якоби и связываем его с гессианом. В качестве следствия из структуры пучков в вариационной последовательности мы получаем, что калибровочно-естественная вторая вариация равна (с точностью до полных дивергенций) вертикальному дифференциалу морфизма Эйлера-Лагранжа. Таким образом, мы воспроизводим для калибровочно-естественных теорий поля высшего порядка хорошо известный (в случае первого порядка) факт, заключающийся в том, что вторая вариация совпадает с гессиановым морфизмом с точностью до полных дивергенций (см., например, работы [1], [26]). В разделе 4 мы характеризуем теоремы Нётер в случае калибровочно-естественных теорий и вводим морфизм Бергманна-Бианки. Как следствие второй теоремы Нётер, существует ковариантный сохраняющийся ток, связанный с лагранжианом, полученным с помощью свертки морфизма ЭйлераЛагранжа с определенным подходящим образом калибровочно-естественным векторным полем Якоби. Мы покажем, что гамильтоновы уравнения для соответствующей гамильтоновой связности (эквивалентные ядру морфизма Эйлера-Лагранжа упомянутого выше лагранжиана) тождественно удовлетворяются, и их можно подходящим образом интерпретировать как обобщенные тождества Бергманна-Бианки и, таким образом, охарактеризовать в терминах обращения в нуль гессиана.

\section{2. СТРУИ КОНЕЧНОГО ПОРЯДКА КАЛИБРОВОЧНО-ЕСТЕСТВЕННЫХ РАССЛОЕНИЙ}

Напомним некоторые основные факты о пространстве струй [3], [8]. Мы работаем с расслоенным многообразием $\pi: \mathbf{Y} \rightarrow \mathbf{X}$, где $\operatorname{dim} \mathbf{X}=n$ и $\operatorname{dim} \mathbf{Y}=n+m$.

Для целых $s \geqslant q \geqslant 0$ мы имеем дело с пространством $s$-струй $J_{s} \mathbf{Y} s$-струйных продолжений (локальных) сечений многообразия $\pi$; в частности, положим $J_{0} \mathbf{Y} \equiv \mathbf{Y}$. Напомним естественные слоения $\pi_{q}^{s}: J_{s} \mathbf{Y} \rightarrow J_{q} \mathbf{Y}, s \geqslant q, \pi^{s}: J_{s} \mathbf{Y} \rightarrow \mathbf{X}$, и среди них аффинные слоения $\pi_{s-1}^{s}$. Обозначим через $V \mathbf{Y}$ векторное подрасслоение касательного расслоения $T \mathbf{Y}$ векторов на $\mathbf{Y}$, вертикальных относительно слоения $\pi$.

Греческие индексы $\sigma, \mu, \ldots$ пробегают значения от 1 до $n$ и обозначают координаты на базе, а латинские индексы $i, j, \ldots$ пробегают значения от 1 до $m$ и обозначают координаты в слое, если не оговорено другое. Обозначим мультииндексы размерности $n$ жирными греческими буквами так, что $\boldsymbol{\alpha}=\left(\alpha_{1}, \ldots, \alpha_{n}\right)$, где $0 \leqslant \alpha_{\mu}, \mu=1, \ldots, n$; злоупотребляя обозначениями, обозначим через $\sigma$ мультииндекс такой, что $\alpha_{\mu}=0$, если $\mu \neq \sigma$, и $\alpha_{\mu}=1$, если $\mu=\sigma$. Кроме того, положим 
$|\boldsymbol{\alpha}|:=\alpha_{1}+\cdots+\alpha_{n}$ и $\boldsymbol{\alpha} !:=\alpha_{1} ! \ldots \alpha_{n}$ !. Карты, индуцированные на $J_{s} \mathbf{Y}$, обозначим как $\left(x^{\sigma}, y_{\boldsymbol{\alpha}}^{i}\right)$, где $0 \leqslant|\boldsymbol{\alpha}| \leqslant s$; в частности, положим $y_{0}^{i} \equiv y^{i}$. Локальные векторные поля и формы $J_{s} \mathbf{Y}$, индуцированные перечисленными выше координатами, обозначим соответственно как $\left(\partial_{i}^{\boldsymbol{\alpha}}\right)$ и $\left(d_{\boldsymbol{\alpha}}^{i}\right)$.

Для $s \geqslant 1$ рассмотрим следующее естественное расщепление, индуцированное естественной контактной структурой на расслоениях струй:

$$
J_{s} \mathbf{Y} \underset{J_{s-1} \mathbf{Y}}{\times} T^{*} J_{s-1} \mathbf{Y}=\left(J_{s} \mathbf{Y} \underset{J_{s-1} \mathbf{Y}}{\times} T^{*} \mathbf{X}\right) \oplus \mathcal{C}_{s-1}^{*}[\mathbf{Y}]
$$

где $\mathcal{C}_{s-1}^{*}[\mathbf{Y}]:=\operatorname{im} \vartheta_{s}^{*}$ и $\vartheta_{s}^{*}: J_{s} \mathbf{Y} \underset{J_{s-1} \mathbf{Y}}{\times} V^{*} J_{s-1} \mathbf{Y} \rightarrow J_{s} \mathbf{Y} \underset{J_{s-1} \mathbf{Y}}{\times} T^{*} J_{s-1} \mathbf{Y}$.

Если $f: J_{s} \mathbf{Y} \rightarrow \mathbb{R}-$ функция, то полагаем $D_{\sigma} f:=\mathcal{D}_{\sigma} f, D_{\boldsymbol{\alpha}+\sigma} f:=D_{\sigma} D_{\boldsymbol{\alpha}} f$, где $D_{\sigma}$ - стандартная полная производная. Для заданного векторного поля $\Xi: J_{s} \mathbf{Y} \rightarrow$ $T J_{s} \mathbf{Y}$ расщепление (1) дает $\Xi \circ \pi_{s}^{s+1}=\Xi_{\mathrm{H}}+\Xi_{\mathrm{V}}$. Мы будем называть $\Xi_{\mathrm{H}}$ и $\Xi_{\mathrm{V}}$ соответственно горизонтальной и вертикальной частями $\Xi$.

Расщепление (1) также индуцирует разложение внешнего дифференциала на $\mathbf{Y}$, $\left(\pi_{s-1}^{s}\right)^{*} \circ d=d_{\mathrm{H}}+d_{\mathrm{V}}$, где $d_{\mathrm{H}}$ и $d_{\mathrm{V}}$ определяются как горизонтальный и вертикальный дифференциальь. Действие $d_{\mathrm{H}}$ и $d_{\mathrm{V}}$ на функции и 1-формы на $J_{s} \mathbf{Y}$ однозначно определяет $d_{\mathrm{H}}$ и $d_{\mathrm{V}}$ (подробности см., например, в работах [8], [27]). Такие разложения всегда повышают порядок объектов.

2.1. Калибровочно-естественные расслоения. Пусть $\mathbf{P} \rightarrow \mathbf{X}$ - главное расслоение со структурной группой $\mathbf{G}$. Пусть $r \leqslant k$-целое, a $\mathbf{W}^{(r, k)} \mathbf{P}:=J_{r} \mathbf{P} \times \underset{\mathbf{X}}{\times} L_{k}(\mathbf{X})$, где $L_{k}(\mathbf{X})$ - расслоение $k$-реперов в $\mathbf{X}$ [3], [28], $\mathbf{W}_{n}^{(r, k)} \mathbf{G}:=\mathbf{G}_{n}^{r} \odot G L_{k}(n)-n о л y$ прямое произведение по отношению к действию $G L_{k}(n)$ на $\mathbf{G}_{n}^{r}$, задаваемое композицией струй, а $G L_{k}(n)$ - группа $k$-реперов в $\mathbb{R}^{n}$. Обозначим через $\mathbf{G}_{n}^{r}$ пространство $(r, n)$-скоростей на $\mathbf{G}[3]$. Расслоение $\mathbf{W}^{(r, k)} \mathbf{P}$ представляет собой главное расслоение над $\mathbf{X}$ со структурной группой $\mathbf{W}_{n}^{(r, k)} \mathbf{G}$. Пусть $\mathbf{F}$ - любое многообразие, а $\zeta: \mathbf{W}_{n}^{(r, k)} \mathbf{G} \times \mathbf{F} \rightarrow \mathbf{F}$ - левое действие $\mathbf{W}_{n}^{(r, k)} \mathbf{G}$ на $\mathbf{F}$. Имеется естественным образом определенное правое действие $\mathbf{W}_{n}^{(r, k)} \mathbf{G}$ на $\mathbf{W}^{(r, k)} \mathbf{P} \times \mathbf{F}$, так что с $\mathbf{W}^{(r, k)} \mathbf{P}$ можно стандартным образом ассоциировать расслоение $\mathbf{Y}_{\zeta}:=\mathbf{W}^{(r, k)} \mathbf{P} \times{ }_{\zeta} \mathbf{F}$ с данной базой $\mathbf{X}$.

ОПРЕДЕЛЕНИЕ 1. Будем говорить, что $\left(\mathbf{Y}_{\zeta}, \mathbf{X}, \pi_{\zeta} ; \mathbf{F}, \mathbf{G}\right)$ является калибровочноестественным расслоением порядка $(r, k)$, ассоциированным с главным расслоением $\mathbf{W}^{(r, k)} \mathbf{P}$ посредством левого действия $\zeta$ группы $\mathbf{W}^{(r, k)} \mathbf{G}$ на многообразии $\mathbf{F}$ [3], [28].

Иногда мы будем опускать нижний индекс $\zeta$, так что все наши рассмотрения будут относиться к $\mathbf{Y}$ как к калибровочно-естественному расслоению, определенному выше.

ЗАмечание 1. Главный автоморфизм $\Phi$ расслоения $\mathbf{W}^{(r, k)} \mathbf{P}$ индуцирует автоморфизм калибровочно-естественного расслоения как

$$
\Phi_{\zeta}: \mathbf{Y}_{\zeta} \rightarrow \mathbf{Y}_{\zeta}:\left[\left(j_{r}^{x} \gamma, j_{k}^{0} t\right), \hat{f}\right]_{\zeta} \mapsto\left[\Phi\left(j_{r}^{x} \gamma, j_{k}^{0} t\right), \hat{f}\right]_{\zeta}
$$


где $\hat{f} \in \mathbf{F}$ и $[\cdot, \cdot]_{\zeta}-$ класс эквивалентности, индуцированый действием $\zeta$.

ОпРЕДЕЛЕНИЕ 2. Определим векторное расслоение над $\mathbf{X}$ правоинвариантных инфинитезимальных автоморфизмов расслоения $\mathbf{P}$, полагая $\mathcal{A}=T \mathbf{P} / \mathbf{G}$.

Также определим векторное расслоение над $\mathbf{X}$ правоинвариантных инфинитезимальных автоморфизмов расслоения $\mathbf{W}^{(r, k)} \mathbf{P}$, полагая $\mathcal{A}^{(r, k)}:=T \mathbf{W}^{(r, k)} \mathbf{P} / \mathbf{W}^{(r, k)} \mathbf{G}$ $(r \leqslant k)$.

ПримеР. Ассоциированное расслоение $\mathbf{W}^{(1,1)} \mathbf{P} \times{ }_{\mu}\left(\mathbb{R}^{n} \oplus \mathfrak{g}\right)$, где $\mu$ - естественное действие $\mathbf{G}_{n}^{1} \odot G L(n)$ над $\left(\mathbb{R}^{n} \oplus \mathfrak{g}\right)$, представляет собой калибровочно-естественное расслоение порядка $(1,1)$. Заданный автоморфизм главного расслоения $\mathbf{P}$ действует на $\mathbf{W}^{(1,1)} \mathbf{P} \times{ }_{\mu} \mathbb{R}^{n} \oplus \mathfrak{g}$ посредством канонического действия группы $\operatorname{Aut}(\mathbf{P})$. Глобальные сечения расслоения $\mathbf{W}^{(1,1)} \mathbf{P} \times_{\mu}\left(\mathbb{R}^{n} \oplus \mathfrak{g}\right)$ находятся во взаимно однозначном соответствии с правоинвариантными инфинитезимальными автоморфизмами расслоения $\mathbf{P}$. Действительно, легко показать, что $\mathbf{W}^{(1,1)} \mathbf{P} \times{ }_{\mu}\left(\mathbb{R}^{n} \oplus \mathfrak{g}\right)$ есть векторное расслоение, и что имеет место изоморфизм $\mathbf{W}^{(1,1)} \mathbf{P} \times_{\mu}\left(\mathbb{R}^{n} \oplus \mathfrak{g}\right) \simeq \mathcal{A}$.

Обозначим через $\mathcal{T}_{\mathbf{X}}$ и $\mathcal{A}^{(r, k)}$ соответственно пучок векторных полей на $\mathbf{X}$ и пучок правоинвариантных векторных полей на $\mathbf{W}^{(r, k)} \mathbf{P}$. Функториальное отображение $\mathfrak{G}$ определяется таким образом, что оно поднимает любой правоинвариантный локальный автоморфизм $(\Phi, \phi)$ главного расслоения $W^{(r, k)} \mathbf{P}$ до единственного локального автоморфизма $\left(\Phi_{\zeta}, \phi\right)$ ассоциированного расслоения $\mathbf{Y}_{\zeta}$. Его инфинитезимальная версия ассоциирует с любым $\Xi \in \mathcal{A}^{(r, k)}$, проектируемым над $\xi \in \mathcal{T}_{\mathbf{X}}$, единственное проектируемое векторное поле $\hat{\Xi}:=\mathfrak{G}(\bar{\Xi})$ на $\mathbf{Y}_{\zeta}-$ калибровочно-естественныи подгем - следующим образом:

$$
\mathfrak{G}: \mathbf{Y}_{\zeta} \underset{\mathbf{X}}{\times} \mathcal{A}^{(r, k)} \rightarrow T \mathbf{Y}_{\zeta}:(\mathbf{y}, \bar{\Xi}) \mapsto \widehat{\Xi}(\mathbf{y})
$$

где для любого $\mathbf{y} \in \mathbf{Y}_{\zeta}$ полагаем

$$
\widehat{\Xi}(\mathbf{y})=\frac{d}{d t}\left[\left(\Phi_{\zeta t}\right)(\mathbf{y})\right]_{t=0},
$$

a $\Phi_{\zeta t}$ обозначает (локальный) поток, соответствующий калибровочно-естественному подъему автоморфизма $\Phi_{t}$.

Это отображение удовлетворяет следующим свойствам [3]:

1) $\mathfrak{G}$ линейно над $i d_{\mathbf{Y}_{\zeta}}$;

2) $T \pi_{\zeta} \circ \mathfrak{G}=i d_{T \mathbf{X}} \circ \bar{\pi}^{(r, k)}$, где $\bar{\pi}^{(r, k)}$ - естественная проекция $\mathbf{Y}_{\zeta} \underset{\mathbf{x}}{\times} \mathcal{A}^{(r, k)} \rightarrow T \mathbf{X}$;

3) для любой пары $(\bar{\Lambda}, \bar{\Xi}) \in \mathcal{A}^{(r, k)}$ имеем $\mathfrak{G}([\bar{\Lambda}, \bar{\Xi}])=[\mathfrak{G}(\bar{\Lambda}), \mathfrak{G}(\bar{\Xi})]$;

4) имеем координатное выражение отображения $\mathfrak{G}: \mathfrak{G}=d^{\mu} \otimes \partial_{\mu}+d_{\nu}^{A} \otimes\left(\mathcal{Z}_{A}^{i \nu} \partial_{i}\right)+$ $d_{\boldsymbol{\lambda}}^{\nu} \otimes\left(\mathcal{Z}_{\nu}^{i \boldsymbol{\lambda}} \partial_{i}\right)$, где $0<|\boldsymbol{\nu}|<k, 1<|\boldsymbol{\lambda}|<r$, а $\mathcal{Z}_{A}^{i \boldsymbol{\nu}}, \mathcal{Z}_{\nu}^{i \boldsymbol{\lambda}} \in C^{\infty}\left(\mathbf{Y}_{\zeta}\right)-$ подходящие функции, зависящие от расслоения.

\section{2. Производные Ли сечений калибровочно-естественных расслоений.}

ОПредЕЛЕНиЕ 3 . Пусть $\gamma$ - (локальное) сечение $\mathbf{Y}_{\zeta}, \bar{\Xi} \in \mathcal{A}^{(r, k)}$, а $\widehat{\Xi}$ - его калибровочно-естественный подъем. Следуя книге [3], определим обобщенную производную 
Ли сечения $\gamma$ вдоль векторного поля $\widehat{\Xi}$ таким образом, что она является (локальным) сечением $£_{\bar{\Xi}} \gamma: \mathbf{X} \rightarrow V \mathbf{Y}_{\zeta}$, задаваемым выражением $£_{\bar{\Xi}} \gamma=T \gamma \circ \xi-\widehat{\Xi} \circ \gamma$.

ЗАмЕчАниЕ 2. Оператор производной Ли, действующий на сечениях калибровочно-естественных расслоений, удовлетворяет следующим свойствам:

1) он является гомоморфизмом алгебр Ли;

$2)$ для любого векторного поля $\bar{\Xi} \in \mathcal{A}^{(r, k)}$ отображение $\gamma \mapsto £_{\bar{\Xi}} \gamma$ есть квазилинейный дифференциальный оператор первого порядка;

3 для любого локального сечения $\gamma$ расслоения $\mathbf{Y}_{\zeta}$ отображение $\bar{\Xi} \mapsto £_{\bar{\Xi}} \gamma$ является линейным дифференциальным оператором;

4) можно рассматривать $£_{\bar{\Xi}}: J_{1} \mathbf{Y}_{\zeta} \rightarrow V \mathbf{Y}_{\zeta}$ как морфизм над базой $\mathbf{X}$. Используя канонические изоморфизмы $V J_{s} \mathbf{Y}_{\zeta} \simeq J_{s} V \mathbf{Y}_{\zeta}$ для всех $s$, имеем $£_{\Xi}\left[j_{s} \gamma\right]=j_{s}\left[£_{\Xi} \gamma\right]$ для любого (локального) сечения $\gamma$ расслоения $\mathbf{Y}_{\zeta}$ и для любого (локального) векторного поля $\Xi \in \mathcal{A}^{(r, k)}$. Более того, для калибровочно-естественных подъемов выполнено фундаментальное соотношение

$$
\widehat{\Xi}_{\mathrm{V}}:=\mathfrak{G}(\bar{\Xi})_{\mathrm{V}}=-£_{\bar{\Xi}} \text {. }
$$

\section{3. ВАРИАЦИОННЫЕ ПОСЛЕДОВАТЕЛЬНОСТИ И ТЕОРЕМЫ НЁТЕР}

Для $s \geqslant 0$ рассмотрим стандартные пучки $\Lambda_{s}^{p} p$-форм на $J_{s} \mathbf{Y}$. При $0 \leqslant q \leqslant s$ рассмотрим пучки $\mathcal{H}_{(s, q)}^{p}$ и $\mathcal{H}_{s}^{p}$ горизонтальных форм относительно соответствующих проекций $\pi_{q}^{s}$ и $\pi_{0}^{s}$. При $0 \leqslant q<s$ рассмотрим подпучки $\mathcal{C}_{(s, q)}^{p} \subset \mathcal{H}_{(s, q)}^{p}$ и $\mathcal{C}^{p}{ }_{s} \subset \mathcal{C}_{(s+1, s)}^{p}$ контактных форм, т.е. горизонтальных форм со значениями в $\mathcal{C}_{s}^{*}[\mathbf{Y}]$ (они имеют свойство обращаться в нуль вдоль любого сечения калибровочно-естественного расслоения).

Пусть $\alpha \in \mathcal{C}_{s}^{1} \wedge \mathcal{H}_{s+1}^{n, h}$. Тогда имеется единственная пара пучковых морфизмов [27], [29], [30]

$$
E_{\alpha} \in \mathcal{C}_{(2 s, 0)}^{1} \wedge \mathcal{H}_{2 s+1}^{n, h}, \quad F_{\alpha} \in \mathcal{C}_{(2 s, s)}^{1} \wedge \mathcal{H}_{2 s+1}^{n, h}
$$

такая, что $\left(\pi_{s+1}^{2 s+1}\right)^{*} \alpha=E_{\alpha}-F_{\alpha}$, а $F_{\alpha}$ локально имеют вид $F_{\alpha}=d_{\mathrm{H}} p_{\alpha}$, где $p_{\alpha} \in$ $\mathcal{C}_{(2 s-1, s-1)}^{1} \wedge \mathcal{H}^{n-1}{ }_{2 s}$

Теперь введем фундаментальный (для наших собственных нужд) морфизм, обозначаемый как $K_{\eta}$, который был предложен в работе [27] и затем изучался в работе [30].

Пусть $\eta \in \mathcal{C}_{s}^{1} \wedge \mathcal{C}_{(s, 0)}^{1} \wedge \mathcal{H}_{s+1}^{n, h}$; тогда имеется единственный морфизм

$$
K_{\eta} \in \mathcal{C}_{(2 s, s)}^{1} \otimes \mathcal{C}_{(2 s, 0)}^{1} \wedge \mathcal{H}_{2 s+1}^{n, h}
$$

такой, что для всех $\Xi: \mathbf{Y} \rightarrow V \mathbf{Y}, E_{\left.j_{s} \Xi\right\rfloor \eta}=C_{1}^{1}\left(j_{2 s} \Xi \otimes K_{\eta}\right)$, где $C_{1}^{1}$ обозначает тензорную свертку на первом множителе, а 」- внутреннее произведение [27], [30]. Более того, имеется единственная пара пучковых морфизмов

$$
H_{\eta} \in \mathcal{C}_{(2 s, s)}^{1} \wedge \mathcal{C}_{(2 s, 0)}^{1} \wedge \mathcal{H}_{2 s+1}^{n, h}, \quad G_{\eta} \in \mathcal{C}_{(2 s, s)}^{2} \wedge \mathcal{H}_{2 s+1}^{n, h}
$$


такая, что $\left(\pi_{s+1}^{2 s+1}\right)^{*} \eta=H_{\eta}-G_{\eta}$, а $H_{\eta}=A\left(K_{\eta}\right) / 2$, где $A$ обозначает антисимметризацию. Более того, $G_{\eta}$ локалъно дается выражением типа $G_{\eta}=d_{\mathrm{H}} q_{\eta}$, где $q_{\eta} \in \mathcal{C}_{(2 s-1, s-1)}^{2} \wedge \mathcal{H}^{n-1}{ }_{2 s} ;$ поэтому $[\eta]=\left[H_{\eta}\right][27],[30]$.

Согласно работам [27], [31] расслоенное расщепление (1) дает расщепление пучков $\mathcal{H}_{(s+1, s)}^{p}=\bigoplus_{t=0}^{p} \mathcal{C}_{(s+1, s)}^{p-t} \wedge \mathcal{H}_{s+1}^{t}$, которое сужается до включения $\Lambda_{s}^{p} \subset \bigoplus_{t=0}^{p} \mathcal{C}^{p-t}{ }_{s} \wedge$ $\mathcal{H}^{t, h}{ }_{s+1}$, где $\mathcal{H}^{p, h}{ }_{s+1}:=h\left(\Lambda_{s}^{p}\right)$ при $0<p \leqslant n$, а сюръективное отображение $h$ определяется как сужение на $\Lambda_{s}^{p}$ проекции указанного выше расщепления на нетривиальное слагаемое с наибольшим значением $t$. Обозначим через $d \operatorname{ker} h$ пучок, стандартным образом порожденный предпучком $d \operatorname{ker} h$. Положим $\Theta_{s}^{*}:=\operatorname{ker} h+d \operatorname{ker} h$.

В работе [31] было доказано, что вариационная последовательность s-го порядка

$$
0 \longrightarrow \mathbb{R}_{\mathbf{Y}} \longrightarrow \Lambda_{s}^{0} \stackrel{\mathcal{E}_{0}}{\longrightarrow} \Lambda_{s}^{1} / \Theta_{s}^{1} \stackrel{\mathcal{E}_{1}}{\longrightarrow} \Lambda_{s}^{2} / \Theta_{s}^{2} \stackrel{\mathcal{E}_{2}}{\longrightarrow} \ldots \stackrel{\mathcal{E}_{I-1}}{\longrightarrow} \Lambda_{s}^{I} / \Theta_{s}^{I} \stackrel{\mathcal{E}_{I}}{\longrightarrow} \Lambda_{s}^{I+1} \stackrel{d}{\longrightarrow} 0
$$

где целое $I$ зависит от размерности слоев в $\mathbf{Y}$ [31], связанная с расслоенным многообразием $\mathbf{Y} \rightarrow \mathbf{X}$, дает точную резольвенту постоянного пучка $\mathbb{R}_{\mathbf{Y}}$ над $\mathbf{Y}$.

Для практических целей ограничимся рассмотрением введенной в работе [27] усеченной вариационной последовательности

$$
0 \longrightarrow \mathbb{R}_{Y} \longrightarrow \mathcal{V}_{s}^{0} \stackrel{\mathcal{E}_{0}}{\longrightarrow} \mathcal{V}_{s}^{1} \stackrel{\mathcal{E}_{1}}{\longrightarrow} \ldots \stackrel{\mathcal{E}_{n}}{\longrightarrow} \mathcal{V}_{s}^{n+1} \stackrel{\mathcal{E}_{n+1}}{\longrightarrow} \mathcal{E}_{n+1}\left(\mathcal{V}_{s}^{n+1}\right) \stackrel{\mathcal{E}_{n+2}}{\longrightarrow} 0
$$

где пучки $\mathcal{V}_{s}^{p}:=\mathcal{C}_{s}^{p-n} \wedge \mathcal{H}_{s+1}^{n, h} / h(d \operatorname{ker} h)$ при $0 \leqslant p \leqslant n+2$ являются подходящими представлениями соответствующих фактор-пучков в вариационной последовательности посредством пучков сечений тензорных расслоений.

ЗАмечаниЕ 3. Сечение $\lambda \in \mathcal{V}_{s}^{n}$ является в стандартной литературе просто лагранжианом порядка $(s+1)$. Более того, $\mathcal{E}_{n}(\lambda) \in \mathcal{V}_{s}^{n+1}$ совпадает со стандартным морфизмом Эйлера-Лагранжа высшего порядка, ассоциированным с $\lambda$. Пусть $\gamma \in \Lambda_{s}^{n+1}$. Морфизм $H_{h d \gamma} \equiv H_{\left[\mathcal{E}_{n+1}(\gamma)\right]}$, где квадратные скобки обозначают класс эквивалентности, называется обобщенным морфизмом Гельмгольца; его ядро совпадает с условиями Гельмгольца локальной вариационности. Интегрируя морфизм $K_{\eta}$ по частям, получаем подходящее представление обобщенного морбизма Якоби, ассоциированного с $\lambda[20],[21],[25]$.

Стандартная производная Ли расслоенных морфизмов по отношению к проектируемому векторному полю $j_{s} \Xi$ переходит на фактор в вариационной последовательности [32]. Вариационная производная Ли $\mathcal{L}_{j_{s}} \Xi$ действует на классах эквивалентности расслоенных морфизмов, являющихся сечениями фактор-пучков в вариационной последовательности. В частности, имеют место следующие два результата [32], которые мы по очевидным причинам называем первой и второй теоремами Нётер.

Теорема 1. Пусть $[\alpha]=h(\alpha) \in \mathcal{V}_{s}^{n}$. Тогда имеем локально (с точностъю до обратных образов)

$$
\left.\left.\left.\mathcal{L}_{j_{s} \Xi}(h(\alpha))=\Xi_{\mathrm{V}}\right\rfloor \mathcal{E}_{n}(h(\alpha))+d_{\mathrm{H}}\left(j_{2 s} \Xi_{\mathrm{V}}\right\rfloor p_{d_{\mathrm{V}} h(\alpha)}+\xi\right\rfloor h(\alpha)\right) .
$$


Теорема 2. Пусть $\alpha \in \Lambda_{s}^{n+1}$. Тогда имеем глобалъно (с точностью до обратных образов)

$$
\left.\mathcal{L}_{j_{s} \Xi}[\alpha]=\mathcal{E}_{n}\left(j_{s+1} \Xi_{\mathrm{V}}\right\rfloor h(\alpha)\right)+C_{1}^{1}\left(j_{s} \Xi_{\mathrm{V}} \otimes K_{h d \alpha}\right) .
$$

Заметим, что вторая теорема Нётер в том виде, как она сформулирована выше, представлена в терминах морфизма $K_{h d \alpha}$.

\section{1. Сохраняющиеся нётеровские токи.}

ОПредЕЛЕНИЕ 4. Пусть $(\widehat{\Xi}, \xi)$ - проектируемое векторное поле на $\mathbf{Y}_{\zeta}$. Пусть $\lambda \in \mathcal{V}_{s}^{n}$ - обобщенный лагранжиан. Будем говорить, что $\widehat{\Xi}-$ симметрия лагранжи-

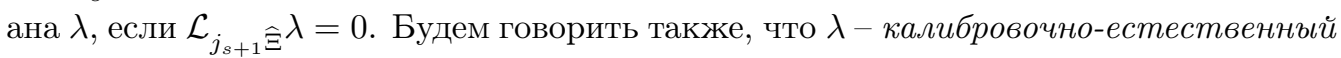
инвариантный лагранжиан, если калибровочно-естественный подъем $(\widehat{\Xi}, \xi)$ любого векторного поля $\bar{\Xi} \in \mathcal{A}^{(r, k)}$ является симметрией для $\lambda$, т.е. если $\mathcal{L}_{j_{s+1}} \bar{\Xi} \lambda=0$. В этом случае проектируемое векторное поле $\widehat{\Xi} \equiv \mathfrak{G}(\bar{\Xi})$ называется калибровочно-естественной симметрией лагранжиана $\lambda$.

Теоремы Нётер принимают весьма частный вид в случае калибровочно-естественных лагранжевых теорий поля (см., например, работы [17], [18]) из-за того, что обобщенная производная Ли сечений калибровочно-естественных расслоений имеет специальные свойства линейности, о которых мы напоминали в п. 2.2, и связана с вертикальной частью калибровочно-естественных подъемов посредством уравнения (4).

ПреДЛОЖенИЕ 1. Пусть $\lambda \in \mathcal{V}_{s}^{n}-$ калибровочно-естественный лагранжиан, $a(\widehat{\Xi}, \xi)$ - калибровочно-естественная симметрия $\lambda$. Тогда $0=-£_{\Xi} \mid \mathcal{E}_{n}(\lambda)+$ $\left.\left.d_{\mathrm{H}}\left(-j_{s} £_{\Xi}\right\rfloor p_{d_{\mathrm{V}} \lambda}+\xi\right\rfloor \lambda\right)$. Предположим, что $\left.\left(j_{2 s+1} \sigma\right)^{*}\left(-£_{\Xi}\right\rfloor \mathcal{E}_{n}(\lambda)\right)=0$. Тогда $(n-$ 1)-борма $\left.\left.\epsilon=-j_{s} £_{\Xi}\right\rfloor p_{d_{\mathrm{V}} \lambda}+\xi\right\rfloor \lambda$ удовлетворяет уравнению $d\left(\left(j_{2 s} \sigma\right)^{*}(\epsilon)\right)=0$.

Если $\sigma$ - критическое сечение для $\mathcal{E}_{n}(\lambda)$, т.е. $\left(j_{2 s+1} \sigma\right)^{*} \mathcal{E}_{n}(\lambda)=0$, то указанное уравнение допускает физическую интерпретацию в виде так называемого слабого закона сохранения для плотности, связанной с $\epsilon$, а про соответствующий пучковый морфизм говорят, что он есть калибровочно-естественный слабо сохраняющийся mok.

3.2. Обобщенные калибровочно-естественные морфизмы Якоби. Пусть $\lambda$ - лагранжиан, а $\widehat{\Xi}_{\mathrm{V}}-$ вариационное векторное поле. Положим $\chi\left(\lambda, \mathfrak{G}(\bar{\Xi})_{\mathrm{V}}\right):=$ $C_{1}^{1}\left(\widehat{\Xi}_{\mathrm{V}} \otimes K_{h d \mathcal{L}_{j_{2 s} \bar{\Xi}_{\mathrm{V}}} \lambda}\right) \equiv E_{\left.j_{s} \widehat{\Xi}\right\rfloor h d \mathcal{L}_{j_{2 s+1} \bar{\Xi}_{\mathrm{V}} \lambda}} \cdot$

ОПРЕДЕЛЕНиЕ 5. Будем называть морфизм $\mathcal{J}\left(\lambda, \mathfrak{G}(\bar{\Xi})_{\mathrm{V}}\right):=E_{\chi\left(\lambda, \mathfrak{G}(\bar{\Xi})_{\mathrm{V}}\right)}$ калибровочно-естественным обобщенным морфизмом Якоби, ассоциированным с лагранжианом $\lambda$ и вариационным векторным полем $\mathfrak{G}(\bar{\Xi})_{\mathrm{V}}$.

Морфизм $\mathcal{J}\left(\lambda, \mathfrak{G}(\bar{\Xi})_{\mathrm{V}}\right)$ является линейным морфизмом по отношению к проекции $J_{4 s} \mathbf{Y}_{\zeta} \underset{\mathbf{X}}{\times} V J_{4 s} \mathcal{A}^{(r, k)} \rightarrow J_{4 s} \mathbf{Y}_{\zeta}$

Пусть $\alpha: J_{s} \mathbf{Y} \rightarrow \stackrel{p}{\wedge} T^{*} J_{s} \mathbf{Y}$ и пусть $L_{j_{s} \Xi_{k}}$-оператор производной Ли, действующий на дифференциальный расслоенный морфизм. 
ОПРЕДЕЛЕНИЕ 6. Пусть $\alpha \in\left(\mathcal{V}_{s}^{n}\right)_{\mathbf{Y}}$ и $\mathcal{L}_{\Xi_{i}}-$ оператор вариационной производной $Л u[32]$ по вариационному векторному полю $\Xi_{i}$. Определим оператор $i$-й вариационной производной следующим образом: $\delta^{i}[\alpha]:=\left[\delta^{i} \alpha\right]=\left[L_{\Xi_{i}} \ldots L_{\Xi_{1}} \alpha\right]=\mathcal{L}_{\Xi_{i}} \ldots \mathcal{L}_{\Xi_{1}}[\alpha]$.

Мы можем следующим образом охарактеризовать гессиан для калибровочно-естественных инвариантных лагранжевых теорий поля в терминах второй вариационной производной.

ОПРЕДЕЛЕНИЕ 7. Определим морфизм

$$
\left.\left.\mathfrak{H}\left(\lambda, \mathfrak{G}(\bar{\Xi})_{\mathrm{V}}\right)=\mathfrak{G}(\bar{\Xi})_{\mathrm{V}}\right\rfloor \mathcal{E}_{n}\left(\mathfrak{G}(\bar{\Xi})_{\mathrm{V}}\right\rfloor \mathcal{E}_{n}(\lambda)\right)
$$

- гессианов морфизм, связанный с $\lambda$ и вариационным векторным полем $\mathfrak{G}(\bar{\Xi})_{\mathrm{V}}(\mathrm{cm}$. также работы [25] и, в частности, [1] по поводу определения гессианова морфизма для теорий поля первого порядка).

ПРЕДЛОЖЕНИЕ 2. Пустъ $\delta_{\mathfrak{G}}^{2} \lambda:=\mathcal{L}_{\mathfrak{G}(\bar{\Xi})_{\mathrm{V}}} \mathcal{L}_{\mathfrak{G}(\bar{\Xi})_{\mathrm{V}}} \lambda-$ вторая вариачионная производная лагранжиана $\lambda$ по вертикальным частям калибровочно-естественных подъемов инфинитезимальных главных автоморфизмов. Тогда

$$
\mathfrak{H}\left(\lambda, \mathfrak{G}(\bar{\Xi})_{\mathrm{V}}\right)=\delta_{\mathfrak{G}}^{2} \lambda .
$$

Действительно,

$$
\left.\left.\left.\mathcal{J}\left(\lambda, \mathfrak{G}(\bar{\Xi})_{\mathrm{V}}\right)=\mathcal{E}_{n}\left(\mathfrak{G}(\bar{\Xi})_{\mathrm{V}}\right\rfloor h(d \delta \lambda)\right)=\mathfrak{G}(\bar{\Xi})_{\mathrm{V}}\right\rfloor \mathcal{E}_{n}\left(\mathfrak{G}(\bar{\Xi})_{\mathrm{V}}\right\rfloor \mathcal{E}_{n}(\lambda)\right)
$$

что можно получить немедленно вследствие того, что $\delta_{\mathfrak{G}}^{2} \lambda=\mathcal{J}\left(\lambda, \mathfrak{G}(\bar{\Xi})_{\mathrm{V}}\right)$, где вторая вариационная производная и обобщенный морфизм Якоби рассматриваются как классы эквивалентности, являющиеся сечениями пучка $\left(\mathcal{V}_{s}^{n}\right)_{\mathbf{Y}_{\zeta} \times V \mathbf{Y}_{\zeta}}$, а горизонтальные дифференциальные контактные формы высших порядков переходят на фактор.

Этот результат обобщает классический результат Голдшмидта и Стернберга [1], связывающий гессиан с морфизмом Якоби для теорий поля первого порядка; кроме того, калибровочно-естественная структура рассматриваемых здесь теорий позволяет определить обобщенный калибровочно-естественный морфизм Якоби, где вариационные векторные поля являются производными Ли сечений калибровочноестественного расслоения по отношению к калибровочно-естественным подъемам.

\section{4. МОРФИЗМ БЕРГМАННА-БИАНКИ}

Рассмотрим теперь член $\omega\left(\lambda, \mathfrak{G}(\bar{\Xi})_{\mathrm{V}}\right):=-£_{\bar{\Xi}} \mid \mathcal{E}_{n}(\lambda)$, появляющийся в формулировке первой теоремы Нётер, приведенной в предложении 1. Подчеркнем, что в общем случае такой член не обращается в нуль вдоль сечений, не являющихся критическими. Путем манипуляций с этим членом ниже мы выведем - при точно определенных условиях - сильно сохраняющиеся токи, т.е. токи, также удовлетворяющие нётеровскому закону сохранения вдоль некритических сечений, рассмотренных впервые в работе [15].

7 Теоретическая и математическая физика, т. 152, № 2, 2007 г. 
Действительно, следуя в основном процедуре, предложенной в работе [15], можно проинтегрировать $\omega\left(\lambda, \mathfrak{G}(\bar{\Xi})_{\mathrm{V}}\right)$ по частям и таким образом определить обобщенныи морфизм Бергманна-Бианки с точностью до горизонтальных дифференциалов.

ОПРЕДЕЛЕНИЕ 8. Будем называть глобальный морфизм $\beta\left(\lambda, \mathfrak{G}(\bar{\Xi})_{\mathrm{V}}\right) \quad:=$ $E_{\omega\left(\lambda, \mathfrak{G}(\bar{\Xi})_{\mathrm{V}}\right)}$ обобщенным морбизмом Бергманна-Бианки, ассоциированным с лагранжианом $\lambda$ и вариационным векторным полем $\mathfrak{G}(\bar{\Xi})_{\mathrm{V}}$.

Координатные выражения для морфизмов $\beta\left(\lambda, \mathfrak{G}(\bar{\Xi})_{\mathrm{V}}\right)$ можно получить с помощью обратной процедуры (см., например, работу [29]). Заметим, в частности, что $\beta\left(\lambda, \mathfrak{G}(\bar{\Xi})_{\mathrm{V}}\right)$ есть не что иное, как морфизм Эйлера-Лагранжа, ассоциированный с новым лагранжианом $\omega\left(\lambda, \mathfrak{G}(\bar{\Xi})_{\mathrm{V}}\right)$, определенным на расслоенном многообразии $J_{2 s} \mathbf{Y}_{\zeta} \times \underset{\mathbf{X}}{\times} J_{2 s} \mathcal{A}^{(r, k)} \rightarrow \mathbf{X} ;$ при этом $\left.\mathfrak{G}(\bar{\Xi})_{\mathrm{V}}\right\rfloor \beta\left(\lambda, \mathfrak{G}(\bar{\Xi})_{\mathrm{V}}\right)$ в точности является гессианом $\mathfrak{H}\left(\lambda, \mathfrak{G}(\bar{\Xi})_{\mathrm{V}}\right)$, связанным с $\lambda$.

Пусть $\mathfrak{K}$ - ядро обобщенного калибровочно-естественного морфизма $\mathcal{J}\left(\lambda, \mathfrak{G}(\bar{\Xi})_{\mathrm{V}}\right)$. В работе [20] мы доказали следующую фундаментальную теорему, которая в общем случае играет существенную роль для характеризации канонических ковариантных сохраняющихся нётеровских токов и, в частности, для доказательства теоремы 4 настоящей работы.

Теорема 3. Обобщенный морбизм Бергманна-Бианки глобалъно обращается в нуль, если и толъко если $\delta_{\mathfrak{G}}^{2} \lambda \equiv \mathcal{J}\left(\lambda, \mathfrak{G}(\bar{\Xi})_{\mathrm{V}}\right)=0$, т.е. если и только если $\mathfrak{G}(\bar{\Xi})_{\mathrm{V}} \in \mathfrak{K}$.

Пусть теперь $\lambda \in \mathcal{V}_{s}^{n}$ - калибровочно-естественный лагранжиан, а $j_{s} \widehat{\Xi}-$ его калибровочно-естественная симметрия, так что $j_{s} \widehat{\Xi}_{\mathrm{V}} \in \mathfrak{K}$. Пусть $D_{\mathrm{H}}-$ внешний дифференциал на расширенном пространстве $J_{2 s} \mathbf{Y}_{\zeta} \underset{\mathbf{X}}{\times} V J_{2 s} \mathcal{A}^{(r, k)}$. При $\beta(\lambda, \mathfrak{K}) \equiv 0$ глобально имеем $\omega(\lambda, \mathfrak{K})=D_{\mathrm{H}} \epsilon(\lambda, \mathfrak{K})$; тогда из первой теоремы Нётер получаем $D_{\mathrm{H}}(\epsilon(\lambda, \mathfrak{K})-\tilde{\epsilon}(\lambda, \mathfrak{K})=0$, что является так называемым калибровочно-естественным "сильным" законом сохранения для глобальной канонической плотности $\epsilon(\lambda, \mathfrak{K})-$ $\tilde{\epsilon}(\lambda, \mathfrak{K})$. Тогда существует канонический глобальный морфизм пучков $\nu(\lambda, \mathfrak{K}) \in$ $\left(\mathcal{V}_{2 s-1}^{n-2}\right)_{\mathbf{Y}_{\zeta} \times \mathfrak{x}}$, так что $D_{\mathrm{H}} \nu(\lambda, \mathfrak{K})=\epsilon(\lambda, \mathfrak{K})-\tilde{\epsilon}(\lambda, \mathfrak{K})$. В силу точности вариационной последовательности существование локального суперпотенциала можно вывести как сечение пучка $\left(\mathcal{V}_{2 s-1}^{n-2}\right)_{\mathbf{Y}_{\zeta} \times \mathcal{A}^{(r, k)}}$. Для физики оказывается существенным, что наш подход дает глобальные сечения редуцированого пучка $\left(\mathcal{V}_{2 s-1}^{n-2}\right)_{\mathbf{Y}_{\zeta} \times \mathfrak{x}}$ без фиксирования какой бы то ни было связности априори. Мы имеем в виду канонические ковариантные токи или соответствующие суперпотенциалы при их сужении на $\mathfrak{K}$.

Как актуальное следствие приведенных выше результатов, видим, что необходимое и достаточное условие того, чтобы калибровочно-естественная симметрия $\widehat{\Xi}$ лагранжиана была генератором канонического ковариантного сильно сохраняющегося тока, состоит в том, что производная Ли сечений калибровочно-естественных расслоений $£_{\Xi}$ должна удовлетворять инвариантным обобщенным уравнениям Якоби (ср. с работой [33]). 
Гамильтоновы уравнения, эквивалентные обобщенным уравнениям

Якоби. Как хорошо известно, вторая теорема Нётер имеет дело со свойствами инвариантности уравнений Эйлера-Лагранжа (так называемыми обобщенными симметриями, или симметриями Бесселя-Хагена, см., например, фундаментальную работу [34]). Хотя симметрии лагранжиана оказываются также симметриями морфизма Эйлера-Лагранжа, обратное, вообще говоря, неверно. Симметрии морфизма Эйлера-Лагранжа, однако, четко связаны со свойствами инвариантности, которыми обладает $\left.\omega\left(\lambda, \mathfrak{G}(\bar{\Xi})_{\mathrm{V}}\right):=-£_{\Xi}\right\rfloor \mathcal{E}_{n}(\lambda)$. Рассмотрим нётеровский закон сохранения, связанный с индуцированными свойствами инвариантности $\omega\left(\lambda, \mathfrak{G}(\bar{\Xi})_{\mathrm{V}}\right)$ (см. также работу [22]).

Заметим, что для всякой симметрии $\bar{\Xi} \in \mathcal{A}^{(r, k)}$ такой, что $\bar{\Xi}_{\mathrm{V}} \in \mathfrak{K}$, имеем

$$
\left.\mathcal{L}_{j_{s} \bar{\Xi}_{H}} \omega(\lambda, \mathfrak{K})=-D_{\mathrm{H}}\left(-j_{s} £_{\bar{\Xi}_{\mathrm{V}}}\right\rfloor p_{D_{\mathrm{V}} \omega(\lambda, \mathfrak{K})}\right) .
$$

Более того, можно утверждать, что $\omega(\lambda, \mathfrak{K})$ обладает свойством естественности, дающим некоторую дополнительную информацию о гамильтоновой структуре калибровочно-естественных теорий поля. Пусть $\bar{\Xi}_{\mathrm{V}} \in \mathfrak{K}$. Имеем $\mathcal{L}_{j_{s}} \bar{\Xi}_{\mathrm{H}} \omega(\lambda, \mathfrak{K})=0$. Следовательно, для любого $\mathfrak{G}(\bar{\Xi})_{\text {н }}$ получаем существование обобщенного сохраняющегося нётеровского тока (который можно было бы интерпретировать как обобщенный тензор энергии-импульса для $\omega(\lambda, \mathfrak{K}))$. Таким образом, если положить $\bar{\Xi}_{\mathrm{V}} \in \mathfrak{K}$, то получим ковариантный закон сохранения

$$
\left.D_{\mathrm{H}}\left(-j_{s} £_{\bar{\Xi}_{\mathrm{V}}}\right\rfloor p_{D_{\mathrm{V}} \omega(\lambda, \mathfrak{K})}\right)=0 .
$$

ОПРЕДЕЛЕНиЕ 9. Определим ковариантую $n$-форму

$$
\left.\mathcal{H}(\lambda, \mathfrak{K})=-j_{s} £\right\rfloor p_{D_{\mathrm{V}} \omega(\lambda, \mathfrak{K})}
$$

как гамильтонову форму для $\omega(\lambda, \mathfrak{K})$ на лежандровом расслоении $\Pi \equiv V^{*}\left(J_{2 s} \mathbf{Y}_{\zeta} \underset{\mathbf{X}}{\times}\right.$ $\left.V J_{2 s} \mathcal{A}^{(r, k)}\right) \wedge\left({ }^{n-1} T^{*} \mathbf{X}\right)$.

Пусть $\Omega$ - мультисимплектическая форма на П. Рассмотрим однородное лежандрово расслоение $\mathbf{Z} \doteq T^{*}\left(J_{2 s}\left(\mathbf{Y}_{\zeta} \underset{\mathbf{X}}{\times} V \mathcal{A}^{(r, k)}\right)\right) \wedge \Lambda^{n-1} T^{*} \mathbf{X}$, снабженное обычной мультисимплектической формой. Хорошо известно, что любое сечение аффинного расслоения $\mathbf{Z} \rightarrow \Pi$ дает гамильтонову форму $\mathcal{H}$ на лежандровом расслоении П с помощью обратного образа канонической внешней $n$-формы $\psi_{J_{2 s}\left(\mathbf{Y}_{\zeta} \times V \mathcal{A}^{(r, k)}\right)}$ такой, что $\Omega=d \psi$. Каждая связность на $J_{2 s}\left(\mathbf{Y}_{\zeta} \underset{\mathbf{X}}{\times} V \mathcal{A}^{(r, k)}\right)$ определяет гамильтонову форму; обратно, каждая гамильтонова форма $\mathcal{H}$ допускает гамильтонову связность $\gamma_{\mathcal{H}}(\lambda, \mathfrak{K})$, для которой $\left.\gamma_{\mathcal{H}}(\lambda, \mathfrak{K})\right\rfloor \Omega=d \mathcal{H}(\lambda, \mathfrak{K})$. Пусть тогда $\gamma_{\mathcal{H}}(\lambda, \mathfrak{K})$ - соответствующая форма гамильтоновой связности [35]. Хорошо известно, что при $\operatorname{dim} \mathbf{X}>1$ имеется, вообще говоря, множество гамильтоновых связностей $\gamma_{\mathcal{H}}$, зависящих от линейной связности на $\mathbf{X}$. В действительности гамильтонова связность $\gamma_{\mathcal{H}}\left(\lambda, \mathfrak{G}(\bar{\Xi})_{\mathrm{V}}\right.$ зависит от импульса $p_{D_{\mathrm{V}} \omega(\lambda, \mathfrak{K})}$. Тем не менее легко видеть, что при $\gamma_{\mathcal{H}}(\lambda, \mathfrak{K})$ горизонтальное распределение, индуцированное подъемом связности $\gamma(\xi)$, однозначно связано с $£_{\Xi}$ согласно обобщенным уравнениям Якоби. 
Приходим к следующей теореме.

ТеоремА 4. Для всех $\mathfrak{G}(\Xi)_{\mathrm{V}} \in \mathfrak{K}$ уравнения Гамильтона для гамильтоновой бормы связности $\gamma_{\mathcal{H}}(\lambda, \mathfrak{K})$ совпадают с ядром обобщенного калибровочно-естественного морфизма Якоби.

Для доказательства теоремы заметим, что уравнения Гамильтона для гамильтоновой связности $\gamma_{\mathcal{H}}(\lambda, \mathfrak{K})$ (эквивалентные ядру морфизма Эйлера-Лагранжа для лагранжиана $\omega(\lambda, \mathfrak{K})$; см., например, книгу [35]) совпадают с $\mathfrak{K}$ в силу теоремы 3 и тем самым удовлетворяются тождественно. Обобщенные тождества БергманнаБианки появляются при этом как связи, обеспечивающие выполнение такой эквивалентности. Вследствие предложения 2 и теоремы 3 они также характеризуются обращением в нуль гессианова морфизма (см. также работу [1] для теорий поля первого порядка).

Благодарности. Работа выполнена при частичной финансовой поддержке GNFM INdAM, MIUR (PRIN 2005) и Туринского университета.

\section{Список литературы}

[1] H. Goldschmidt, S. Sternberg, Ann. Inst. Fourier, 23:1 (1973), 203-267.

[2] А.В. Бочаров, А.М. Вербовецкий, А.М. Виноградови др., Симметрии и законы сохранения уравнений математической физики, Факториал, М., 1997.

[3] I. Kolář, P. W. Michor, J. Slovák, Natural Operations in Differential Geometry, Springer, Berlin, 1993.

[4] D. Krupka, "Some geometric aspects of variational problems in fibred manifolds", Folia Fac. Sci. Nat. Univ. Purk. Brunensis, Physica, 14, J. E. Purkyně Univ., Brno, 1973, 1-65; math-ph/0110005.

[5] B. A. Kuperschmidt, "Geometry of jet bundles and the structure of Lagrangian and Hamiltonian formalism", Geometric Methods in Mathematical Physics, Proc. NSF-CBMS Conf. (Lowell, 1979), Lect. Notes Math., 775, eds. G. Kaiser, J. E. Marsden, Springer, Berlin, 1980, 162-218.

[6] P. J. Olver, Applications of Lie Groups to Differential Equations, 2nd ed., Grad. Texts in Math., 107, Springer, New York, 1993.

[7] R.S. Palais, Foundations of Global Non-linear Analysis, Benjamin, New York-Amsterdam, 1968.

[8] D. J. Saunders, The Geometry of Jet Bundles, London Math. Soc. Lect. Note Ser., 142, Cambridge Univ. Press, Cambridge, 1989.

[9] A. M. Vinogradov, J. Math. Anal. Appl., 100:1 (1984), 1-40; 41-129.

[10] F. Takens, J. Diff. Geom., 14 (1979), 543-562.

[11] W. M. Tulczyjew, Bull. Soc. Math. France, 105 (1977), 419-431.

[12] А. М. Виноградов, ДАН СССР, 236:2 (1977), 284-287.

[13] А. М. Виноградов, ДАН СССР, 238:5 (1978), 1028-1031.

[14] R. Vitolo, Diff. Geom. Appl., 10:3 (1999), 225-255.

[15] P. G. Bergmann, Phys. Rev., 75 (1949), 680-685.

[16] Э. Нётер, "Инвариантные вариационные задачи", Вариационные принципы механики, Физматлит, М., 1959, 604-630.

[17] L. Fatibene, M. Francaviglia, M. Palese, Math. Proc. Cambridge Philos. Soc., 130 (2001), 555-569.

[18] P. Matteucci, Rep. Math. Phys., 52:1 (2003), 115-139. 
[19] J. L. Anderson, P. G. Bergmann, Phys. Rev., 83:5 (1951), 1018-1025.

[20] M. Palese, E. Winterroth, Arch. Math. (Brno), 41:3 (2005), 289-310.

[21] M. Palese, E. Winterroth, Rep. Math. Phys., 54:3 (2004), 349-364.

[22] M. Francaviglia, M. Palese, E. Winterroth, Rep. Math. Phys., 56:1 (2005), 11-22; math-ph/0407054.

[23] M. Francaviglia, M. Palese, E. Winterroth, "Second variational derivative of gauge-natural invariant Lagrangians and conservation laws", Differential Geometry and its Applications, Proc. IX Int. Conf. (Prague, Czech Republic, 2004), eds. J. Bures et al., Matfyzpress, Prague, 2005, 591-604.

[24] M. Palese, E. Winterroth, "Gauge-natural field theories and Noether theorems: canonical covariant conserved currents", Geometry and Physics (Srni, CZ, 2005), Rend. Circ. Mat., Palermo, 2006, 161-174.

[25] M. Francaviglia, M. Palese, R. Vitolo, Diff. Geom. Appl., 22:1 (2005), 105-120.

[26] M. Giaquinta, S. Hildebrandt, Calculus of Variations. Vol. I. The Lagrangian Formalism, Grundlehren Math. Wiss., 310, Springer, Berlin, 1996.

[27] R. Vitolo, Math. Proc. Cambridge Philos. Soc., 125:1 (1999), 321-333.

[28] D. J. Eck, Mem. Amer. Math. Soc., 33:247 (1981), 1-48.

[29] I. Koláŕ, J. Geom. Phys., 1:2 (1984), 127-137.

[30] I. Kolář, R. Vitolo, Math. Proc. Cambridge Philos. Soc., 135:2 (2003), 277-290.

[31] D. Krupka, "Variational sequences on finite order jet spaces", Differential Geometry and its Applications (Brno, Czechoslovakia, 1989), eds. J. Janyška, D. Krupka, World Scientific, Singapore, 1990, 236-254.

[32] M. Francaviglia, M. Palese, R. Vitolo, Czechoslovak Math. J., 52(127):1 (2002), 197-213.

[33] M. Godina, P. Matteucci, Int. J. Geom. Meth. Mod. Phys., 2 (2005), 159-188.

[34] A. Trautman, Commun. Math. Phys., 6 (1967), 248-261.

[35] L. Mangiarotti, G. Sardanashvily, Connections in Classical and Quantum Field Theory, World Scientific, Singapore, 2000. 